\title{
Car Detection in Aerial Images of Dense Urban Areas
}

\author{
Mohamed ElMikaty, Member, IEEE, and Tania Stathaki, Member, IEEE
}

\begin{abstract}
With the ever-increasing demand in the analysis and understanding of aerial images in order to remotely recognise targets, this paper introduces a robust system for the detection and localisation of cars in images captured by air vehicles and satellites. The system adopts a sliding-window approach. It compromises a window-evaluation and a window-classification sub-systems. The performance of the proposed framework was evaluated on the Vaihingen dataset. Results demonstrate its superiority to the state of the art.
\end{abstract}

Index Terms-Car Detection, Automatic Target Recognition, Airborne Imagery.

\section{INTRODUCTION}

$\mathbf{V}$ ISUAL detection of small targets in aerial imagery and the identification of their positions relative to the rest of a given image have attracted many researchers in the fields of image analysis and computer vision over the past years, as it has many useful applications in surveillance, military, remote sensing and other sectors. In this work, we consider cars to be the small-scale targets of interest (contained in $48 \times 48$ pixel area) and the aim is to carry out the task of their detection and localisation in aerial images of typical urban scenes. Such images are usually captured by various types of air vehicles and satellites [1]. This task of detection and localisation is very challenging because of the large intraclass variability that stems from the great differences in the visual appearance among cars, such as differences in colour intensities, size, shape, contours and boundaries. Moreover, there is a strong dependence of the appearance on the viewing angle. In order to overcome this problem, many researchers have focused on the problem of car detection from aerial images, wherein images are taken from a vertical or a slightlyoblique viewpoint. This constrained viewpoint facilitates the detection process and decreases the intra-class variability.

Another major problem that usually hinders the design of a car detection system is the presence of various types of objects other than cars that have very similar rectilinear structures to that of cars, such as buildings' roofs and windows in urban scenes. Furthermore, in some situations parts of different objects appear collectively similar to cars. Consequently, a significant inter-class similarity is expected and the detection problem (class-separation problem) becomes tremendously

Manuscript received October, 2016 and revised April, 2017. This work is funded by Engineering and Physical Sciences Research Council (EPSRC) and BAE SYSTEMS Military Air and Information (UK). The Vaihingen dataset was provided by the German Society for Photogrammetry, Remote Sensing and Geoinformation (DGPF).

M. ElMikaty and T. Stathaki are with the Department of Electrical and Electronic Engineering, Imperial College London, London, SW7 2AZ, UK e-mail: (\{m.elmikaty, t.stathaki\}@imperial.ac.uk). challenging. Accordingly, most past works have suffered from either having low true-positive rates or high false-positive rates.

The primary focus of this work is to design a robust system for the detection and the localisation of cars in aerial images of urban scenes using implicit models as their effectiveness in cluttered environments was corroborated in our previous work [2]. The proposed system can be seen as a combination of two sub-systems. The first sub-system aims to restrict search areas in order to reduce the rate of false positives and increase the number of windows that can be processed per second. This is achieved by evaluating windows based on their likelihood to contain cars. A Gaussian-Mixture-Model (GMM) [3] classifier is exploited to extract regions of interest, more specifically roads and parking areas. In addition, detection windows are filtered based on their colour, gradient and texture properties in a novel way.

The second sub-system exploits an ensemble of descriptors that depicts the distribution of gradients and colours [2]. A support-vector-machine (SVM) classifier is then used to distinguish descriptors that belong to cars.

Among the main contributions that are introduced here are: (i) a novel accurate method to estimate the dominant orientation of detection windows, (ii) automatic extraction of regions of high likelihood to contain targets in order to restrict search areas and (iii) robust detection of cars in urban scenes with high true-positive and low false-positive rates. The proposed framework is different from other works in: (i) the choice and encoding of features, (ii) the way detection windows are filtered, (iii) that it does not depend on the use of pre-saved road maps, (iv) that it uses only one model for both the foreground and the background, unlike some works that use multiple independent models and v) that it achieves a high precision rate using linear classification relative to the state of the art.

The performance of the proposed system was evaluated on the challenging Vaihingen dataset that consists of typical urban scenes with complex structures. Results demonstrate the reliability of the proposed framework in terms of the achieved low false-positive rate and its superiority to the state of the art.

This paper is organised as follows. A review of the literature is given in Sec. II. In Sec. III, the proposed car detection framework is introduced, followed by a brief description of the Vaihingen dataset that was used for training and testing. Results are demonstrated and discussed in Sec. V. Conclusions are drawn in Sec. VI. 


\section{RELATED WORK}

Several methodologies have been proposed to solve the problem of car detection in aerial images from a single viewpoint [4] and several types of images have been investigated, such as colour images, synthetic aperture radar and others [5] [6]. Nevertheless, our focus here is on the detection of cars in colour images and notable related past works will be discussed in this section. Most previously-proposed methodologies can be categorised into four main groups: the first group used explicit models, the second group used implicit models, the third group exploited road maps alongside either an explicit or an implicit model and the fourth group employed deep neural networks.

In explicit models, 2-D and 3-D templates that represent cars are designed and the detection process is carried out by the computation of the cost of matching between the patch under examination and the pre-designed template. Cars are thought to be located in areas, where the cost is below a pre-defined threshold. These models were common among early works because of the unavailability of high-resolution aerial images. Examples of explicit models are the works in [7]-[9], wherein 3-D templates of cars were exploited to characterise the geometric structure of cars' contours and the colours of cars' bodies, windscreens and shadows. In a similar fashion, Choi and Yang [10] adopted a "bottom-up" approach and used a mean-shift clustering algorithm [11] to form blobs of pixels which possess a similar structure to that of cars. Problems of this group of approaches arose from the designed template as researchers, in most cases, had to design generic templates in order to cope with the high intra-class variability. These generic templates were simple and they did not incorporate fine and discriminative image features that distinguish cars. Consequently, they usually fit to multiple locations within the image of interest. In addition, there are common problems that are usually associated with the use of explicit models, as for example, the high computational cost incurred by the matching process and the significant degradation in performance, when they are applied in urban scenes with heavily-cluttered backgrounds [12].

Unlike explicit models, implicit models use different image descriptors that are exploited to capture and encode different cues of targets and a classifier is used to discriminate descriptors that associate with the target of interest from other descriptors. Many works have combined multiple image descriptors aiming to achieve a better description and encoding of the visual properties of cars as it is widely known that using more than a single descriptor has a huge impact on the overall performance of the designed detector. Nevertheless, the performance is significantly affected by the choice of the features and descriptors used and there are not any solid or universal theoretical analyses about this choice in the open literature and only guidelines can be found. Examples of implicit models are the work of Nguyen et al. [12], who used Haar wavelets [13], Histogram of Oriented Gradients (HOG) [14] and Local Binary Patterns (LBP) [15] and the work of Kembhavi et al. [16], who exploited colour probability maps, pairs of pixels and HOG descriptors in an ensemble of image descriptors of 70,000 dimensions. Similarly, Shao et al. [17] used HOG, LBP and opponent histograms in their vehicle detector to form a single descriptor of 6760 dimensions. In a different fashion, Razakarivony and Jurie [18] trained two different classifiers one for the foreground and the other for the background on different features including raw pixel values, gradients and HOG features. Recently, Madhogaria et al. [19] used HOG features to detect cars in a two-stage classification process.

In order to restrict search areas to regions that are likely to contain cars, several previous works have exploited road maps to extract roads and parking areas from the image under examination as an initial step alongside either an explicit or an implicit model. For instance, the work proposed by Moon et al. [20], [21], wherein they utilised the derivative of the doubleexponential filter to extract gradients and the work of Zheng $e t$ al. [22], who used morphological structuring elements and a classifier to distinguish actual car pixels. In a similar manner, Eikvil et al. [23] proposed a detection approach that separates regions with high probability to contain cars, followed by two stages of object classification exploiting multi-spectral images, geometric properties and road networks. Following the same idea, Tuermer et al. [24] proposed filtering areas with very low probability to contain cars using road databases, disparity maps and a pre-processing stage. Whilst using road maps provides better performance in terms of detection and false-positive rates, it requires accurate road maps to be known a priori and a precise map-projection method or the use of a global positioning system (GPS).

Unlike the aforementioned flat models, deep models for the detection of cars in airborne imagery can be found in very few works. They require massively large training datasets to be able to generalise and the acquisition of such aerial datasets is very challenging in terms of time and resources. A representative example is the work of Chen et al. [25], who used a hierarchical deep model in a hybrid fashion. Although their method, achieved high accuracy, their testing dataset contained mainly vast areas with a large number of cars, i.e, parking areas in urban cities. Following the same direction of using deep models, Yi et al. [26] used a pre-trained AlexNet [27] for the detection of cars in WAMI images after modifying the input and the output layers, wherein they converted the problem of the detection of cars in airborne imagery to a twoclass-separation problem, resulting in a poor localisation.

\section{PROPOSED FRAMEWORK}

Regarding a car in a given aerial image, its edge map is expected to consist of several rectilinear structures that correspond to the boundaries of its body and front and rear windscreens, when edges are extracted using common edge detectors, e.g., Canny edge detector [28]. Although these rectilinear structures form together a set of high-curvature points, e.g., corners, which are generally crucial to the detection process, they are incapable of discriminating cars from other objects. For instance, in confounding situations, the contours of non-car objects that have similar edge maps can be easily confused with the contours of cars. This makes 


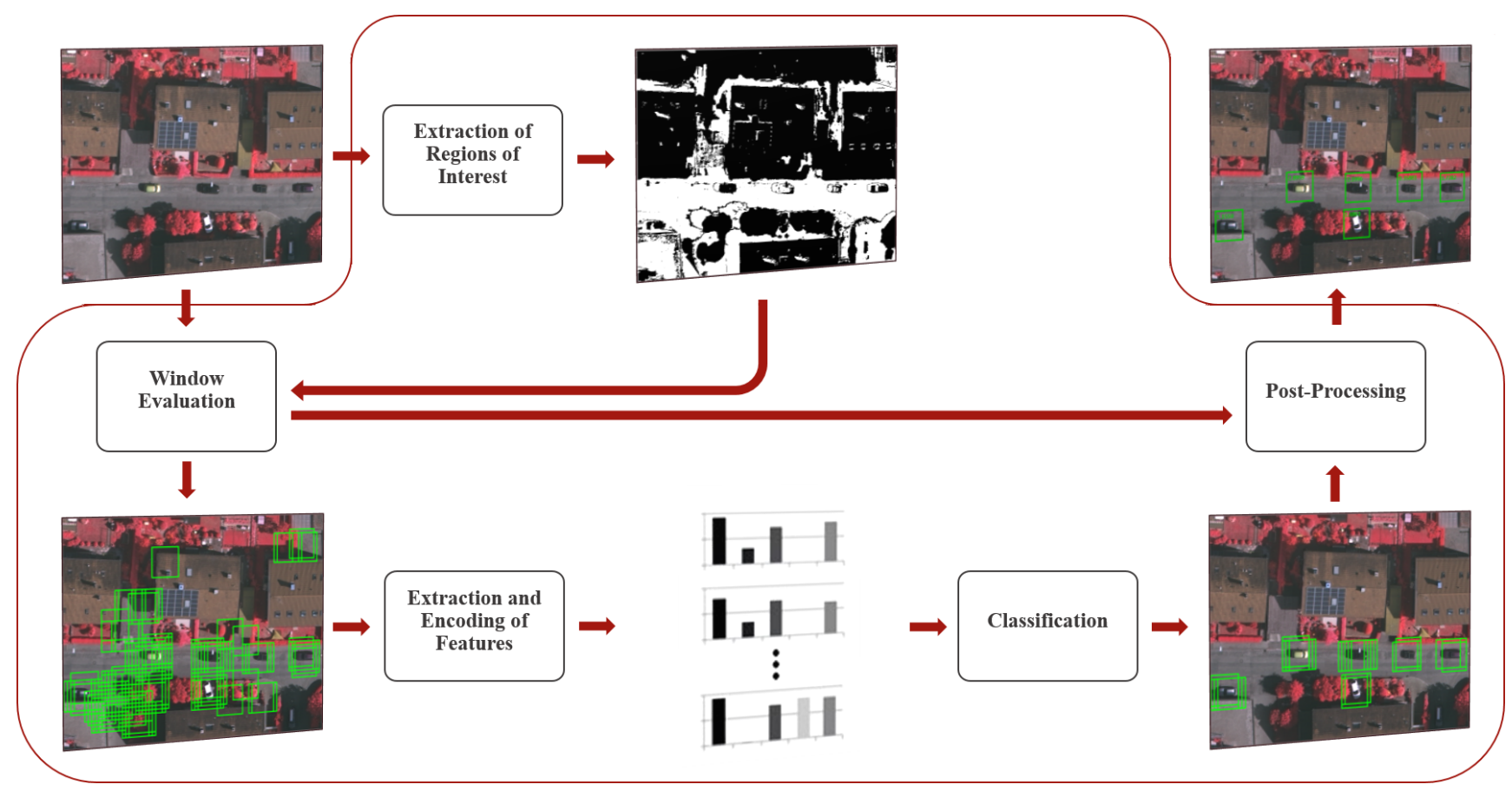

Fig. 1: Data flow in the proposed framework for the detection of cars in aerial images: Top left: input image; top middle: extracted regions of interest represented by a binary mask; top right: processed image. Bottom left: candidate detection windows; bottom middle: schematic representation of the computed feature vectors; bottom right: all claimed detections by the used classifier. Shown images were scaled for viewing.

implicit-model based methods superior to other methods as they can encode different image cues taking various fine details into account. The proposed implicit model exploits two image cues, which are the distribution of gradients and the distribution of colours, as they proved their effectiveness in our previous work [2]. The pipeline of the proposed framework consists of five main stages: window evaluation, extraction and encoding of features, classification and postprocessing as well as extraction of regions of interest, as shown in Fig. 1.

A sliding-window approach is adopted in the present work. A square detection window of $64 \times 64$ pixels and an associated stride length of eight pixels is used as cars in the Vaihingen dataset [29] are of almost similar sizes. The dataset was captured from a relatively high altitude. Each detection window is processed through the pipeline and only successful candidate windows are passed to the following stages.

\section{Choice of Parameters}

Values of certain parameters used in the implementation of the proposed framework were set empirically by testing excessively the properties of a large number of image samples from the training dataset using cross validation. Details of the implementation of the proposed framework are given in the following sections.

\section{A. Extraction of Regions of Interest}

In urban scenes, cars are more likely to be found in road and parking areas [20]-[24]. The extraction of these areas prior to the detection process would eliminate a large number of false positives, which are produced as a result of the visual similarity among cars and other objects. Nonetheless, the method to extract such regions should be simple as a rough estimation is only required, as it will be shown.

Distinctive colour properties that belong to road areas can be extracted using a generative classifier that depends on the statistical properties, e.g., mean and covariance [3]. An example of this type of classifier is the Gaussian Mixture Model (GMM) classifier that aims to fit a family of Gaussians to a given feature distribution. These distinctive properties can be used to segment road areas. In this work, a GMM classifier is used to divide the colour space into several sub-spaces with the aim to extract road and parking areas. Depending on the contribution of the pixel intensity to the three colour channels, a pixel is classified corresponding to road areas if it belongs to the Gaussian members that correspond to the colour subspaces of roads. The probability density function $p d f$ of the colour feature vector $\mathbf{c}$ is modelled here as a mixture of seven Gaussian components. Regions of interest are represented by the closest two components to the mean intensity of road regions. Two components were chosen in order to have a wider range of colour intensities that are close to those of roads and parking areas in order to accommodate for the differences in illumination conditions.

$$
p d f(\mathbf{c})=\sum_{n} \pi_{\mathbf{c}, n} \mathcal{N}\left(\mathbf{c} \mid \mu_{\mathbf{c}, n}, \mathbb{R}_{\mathbf{c c}, n}\right), n \in\{1,2, \ldots, 7\}
$$

where $\mathbf{c}$ is a three-dimensional feature vector, wherein each feature corresponds to the pixel intensity of each colour channel in the RGB colour representation, $\mathcal{N}\left(\mathbf{c} \mid \mu_{\mathbf{c}, n}, \mathbb{R}_{\mathbf{c c}, n}\right)$ is the $n^{\text {th }}$ Gaussian component with mean $\mu_{\mathbf{c}, n}$ and covariance $\mathbb{R}_{\mathbf{c c}, n}$ and $\pi_{\mathbf{c}, n}$ is the mixing coefficient with the following 
constraints:

$$
\left\{\begin{array}{l}
\sum_{n} \pi_{\mathbf{c}, n}=1 \\
0 \leq \pi_{\mathbf{c}, n} \leq 1
\end{array}\right.
$$

A Gaussian component $\mathcal{N}\left(\mathbf{c} \mid \mu_{\mathbf{c}, n}, \mathbb{R}_{\mathbf{c c}, n}\right)$ is a multivariate Gaussian function and can be defined as:

$$
\begin{aligned}
& \mathcal{N}\left(\mathbf{c} \mid \mu_{\mathbf{c}, n}, \mathbb{R}_{\mathbf{c c}, n}\right)= \\
& \frac{1}{(2 \pi)^{D / 2}\left|\mathbb{R}_{\mathbf{c c}, n}\right|^{1 / 2}} \exp \left\{-\frac{1}{2}\left(\mathbf{c}-\mu_{\mathbf{c}, n}\right)^{T} \mathbb{R}_{\mathbf{c c}, n}^{-1}\left(\mathbf{c}-\mu_{\mathbf{c}, n}\right)\right\}
\end{aligned}
$$

In this work, parameters $\mu_{\mathbf{c}, n}, \mathbb{R}_{\mathbf{c c}, n}$ and $\pi_{\mathbf{c}, n}$ were estimated from $K$ training samples using the iterative expectationmaximisation (EM) algorithm [30]. This iterative algorithm consists of two main steps the $E$ step and the $M$ step.

In the $E$ step, current values for $\mu_{\mathbf{c}, n}, \mathbb{R}_{\mathbf{c c}, n}$ and $\pi_{\mathbf{c}, n}$ are used to compute an estimation of the posterior probabilities $\hat{\gamma}_{k, n}$.

$$
\hat{\gamma}_{k, n}=\frac{\pi_{x, n} \mathcal{N}\left(\mathbf{c}_{k} \mid \mu_{\mathbf{c}, n}, \mathbb{R}_{\mathbf{c c}, n}\right)}{\sum_{j} \pi_{\mathbf{c}, j} \mathcal{N}\left(\mathbf{c}_{k} \mid \mu_{\mathbf{c}, j}, \mathbb{R}_{\mathbf{c c}, j}\right)}, j \in\{1,2, \ldots 7\}
$$

In the $M$ step, parameters $\mu_{\mathbf{c}, n}, \mathbb{R}_{\mathbf{c c}, n}$ and $\pi_{\mathbf{c}, n}$ are recomputed based on the estimated $\hat{\gamma}_{k, n}$ :

$$
\begin{gathered}
\Gamma_{n}=\sum_{k} \hat{\gamma}_{k, n}, k \in\{1,2,, \ldots K\} \\
\hat{\mu}_{\mathbf{c}, n}=\frac{1}{\Gamma_{n}} \sum_{k} \hat{\gamma}_{k, n} \mathbf{c}_{k}, k \in\{1,2, \ldots K\} \\
\hat{\mathbb{R}}_{\mathbf{c}, n}=\frac{1}{\Gamma_{n}} \sum_{k} \hat{\gamma}_{k, n}\left(\mathbf{c}_{k}-\hat{\mu}_{\mathbf{c}, n}\right)\left(\mathbf{c}_{k}-\hat{\mu}_{\mathbf{c}, n}\right)^{T}, k \in\{1,2,, \ldots K\} \\
\hat{\pi}_{\mathbf{c}, n}=\frac{\Gamma_{n}}{K}
\end{gathered}
$$

The log likelihood is then computed using Equ. 9 and the above procedure is iterated till convergence is achieved.

$$
\begin{aligned}
\ln p d f\left(\mathbf{C} \mid \mu_{\mathbf{C}}, \mathbb{R}_{\mathbf{C C}}, \pi\right) & =\sum_{k} \ln \left\{\sum_{n} \pi_{n} \mathcal{N}\left(\mathbf{c}_{k} \mid \mu_{\mathbf{c}, n}, \mathbb{R}_{\mathbf{c c}, n}\right)\right\} \\
k & \in\{1,2, \ldots, K\}, n \in\{1,2, \ldots, 7\}
\end{aligned}
$$

A new data sample is assigned to one of the Gaussian components according to the maximum posterior probability $\hat{\gamma}_{k, n}$. More details about the GMM classifier and the EM algorithm can be found in [3], [30]. A binary mask that indicates whether this given pixel corresponds to the colour sub-space of roads will be then created. The produced binary mask is passed to the window-evaluation stage, where the likelihood of a given detection window will be partially assessed based on the amount of overlap with the created binary mask.

\section{B. Window Evaluation}

To reduce search areas, detection windows that are unlikely to contain cars are eliminated based on several cues to increase the number of windows that can be processed per second. The elimination process of detection windows that are unlikely to be placed around cars is based on the amount of overlap with the created binary mask that represents the regions of interest as well as a novel three-step filtering process. The output of this stage is a number of candidate detection windows and is integrated in both the extraction-and-encoding-of-features and the post-processing stages.

The overlap between a given detection window and the created binary mask is computed and its amount controls how detection windows are eliminated as follows: (i) detection windows that have an overlap less than $20 \%$ are discarded, (ii) detection windows that have an overlap more than $20 \%$ and have relatively high confidence scores are kept, (iii) detection windows with low confidence scores and an overlap of more than $30 \%$ are considered as true detections and (iv) detection windows with low confidence scores and not enough amount of overlapping are discarded. Here the confidence score is used to resemble the distance from the separating hyper-plane that is decided using the Support Vector Machine (SVM) classifier, as it will be discussed later. The intuition is cars occupy approximately $56 \%$ of the detection window because they are contained in patches of size $48 \times 48$ pixels, whereas the detection window size id $64 \times 64$ pixels. The rest of the detection window covers either roads solely or road areas on one side of the car and on the other side irrelevant objects. This yields to the conclusion that the percentage of the pixels that corresponds to road areas is at least $20 \%$. Therefore, condition (i) is a general condition for all detection windows regardless of the associated confidence score and it can be examined prior to computing image descriptors. The other three conditions are examined after the classification process and hence, they are integrated in the post-processing stage.

A three-step filtering process is then carried out on detection windows to measure, in a novel way, their objectness [31] and their likelihood to contain a target.

First, the variation in the distribution of grey levels across a given detection window is examined by the computation of two 32-bin grey-level histograms $\mathbf{h}_{w}$ and $\mathbf{h}_{c}$, one for the whole detection window and the other for the $32 \times 32$ central square area. If the similarity between the two histograms is more than $50 \%$, the window is discarded as this highly indicates that it belongs to a flat area with a constant or a slowlyvarying colour distribution, such as empty roads and vegetation areas. This check is performed using the Manhattan distance $r_{\text {Manhattan }}=\sum_{k=1}^{32}\left|\mathbf{h}_{w}(k)-\mathbf{h}_{c}(k)\right|$ between those two colour histograms with a threshold value of 2048 as the size of the detection window is $64 \times 64$ pixels.

Second, the texture of the greyscale detection window is examined locally in a new fashion. Pixels of each local cell of $8 \times 8$ pixels are divided into four sub-cells of $4 \times 4$. Pixels of each sub-cell are further divided into two groups according to whether the pixel value is larger than the average intensity value of the sub-cell or smaller. The difference between the average intensities of the two groups is calculated. The cell is then labeled with the mean value of the differences in average intensities of the four constituting sub-cells. If the standard deviation $\sigma_{\text {texture }}$ of the responses of the computed mean values is larger than 32 , the detection window is not considered for further processing. The intuition behind this is that a high 
standard deviation of the mean values of the differences in average intensities (more than one eighth of the intensity range $[0,255])$ indicates a highly-textured surface that cannot belong to cars as the surfaces of cars tend to be smooth with low amount of texture.

Third, the orientation of gradients is investigated. For a given image $\mathbf{I}$, magnitudes of intensity gradients $\mathbf{M}_{g}(x, y)$ at each pixel and their orientations $\mathbf{O}_{g}(x, y)$ are calculated over the detection window. This is done by the computation of the first-order discrete derivatives $\frac{\partial \mathbf{I}}{\partial x}$ and $\frac{\partial \mathbf{I}}{\partial y}$ of $\mathbf{I}$ using a nonsmoothing filter of [- $\left.\begin{array}{lll}-1 & 0 & 1\end{array}\right]$ in both vertical and horizontal directions for each colour channel and the maximum gradient is kept with its associated orientation:

$$
\begin{gathered}
\mathbf{M}_{k}(x, y)=\sqrt{\left(\frac{\partial \mathbf{I}_{k}(x, y)}{\partial x}\right)^{2}+\left(\frac{\partial \mathbf{I}_{k}(x, y)}{\partial y}\right)^{2}}, k \in\{1,2,3\} \\
\mathbf{M}_{g}(x, y)=\max _{k \in\{1,2,3\}}\left[\mathbf{M}_{k}(x, y)\right] \\
\mathbf{O}_{g}(x, y)=\arctan \left(\frac{\partial \mathbf{I}_{k}(x, y)}{\partial y} / \frac{\partial \mathbf{I}_{k}(x, y)}{\partial x}\right) \mid \mathbf{M}_{g}(x, y) \\
=\max _{k \in\{1,2,3\}}\left[\mathbf{M}_{k}(x, y)\right]
\end{gathered}
$$

Then, the magnitudes of gradients of local cells of $4 \times 4$ pixels are accumulated independently in nine-bin histograms $\mathbf{g}_{\text {cell }}$ according to their unsigned orientations:

$$
\mathbf{g}_{\text {cell }}(k)=\sum_{x, y} \mathbf{M}_{g}(x, y) \mid \mathbf{O}_{g}(x, y) \in \mathbf{o}_{k}, k \in\{1,2, \ldots, 9\}
$$

where $\mathbf{o}_{k}$ is the $k^{t h}$ bin of orientation interval of width $20^{\circ}$. For a typical detection window containing a car, there should be a dominant orientation of the gradients that corresponds to the direction that is perpendicular to the orientation of that car. To examine this property, the covariance matrix $\mathbb{R}_{\mathbf{g g}}=\mathbb{E}\left\{\mathbf{g g}^{H}\right\}$ of the $L 2$-normalised responses $\mathbf{g}_{\text {cell }} \leftarrow \mathbf{g}_{\text {cell }} / \sqrt{\left\|\mathbf{g}_{\text {cell }}\right\|_{2}^{2}+\epsilon^{2}}$ from local cells, where $\epsilon$ is a small constant and is equal to 0.01 , is computed. Then, it is eigen-decomposed. If the maximum eigenvalue $d_{\max }$ is less than 0.09 , the window is discarded. The idea is that the second order statistics of the received signal given by its covariance matrix $\mathbb{R}_{\mathrm{gg}}$ can be used to determine the number of the dominant features and their positions within the feature vector using the rank of $\mathbb{R}_{\mathrm{gg}}$ and the order of their significant eigenvalues $d_{i}$, respectively. This three-step process of window filtering helps reduce the search areas and consequently, it increases the number of detection windows that can be processed per second.

Estimation of the Orientations of Cars: The orientation of cars $O_{c a r}$ is discretised into nine orientations at $10^{\circ}, 30^{\circ}, 50^{\circ}$, $70^{\circ}, 90^{\circ}, 110^{\circ}, 130^{\circ}, 150^{\circ}$ and $170^{\circ}$. It is estimated to be in a perpendicular orientation to the bin orientation corresponding to maximum eigenvalue $d_{\max }$ of the covariance matrix $\mathbb{R}_{\mathrm{gg}}$ of locally-accumulated gradients computed over $4 \times 4$ cells in the window-evaluation stage:

$$
O_{d_{\max }}=(10+20(k-1))^{\circ} \mid d_{k}=\max _{k \in\{1,2, \ldots, 9\}}\left[\operatorname{eig}\left(\mathbb{R}_{\mathbf{g g}}\right)\right]
$$

\section{Extraction and Encoding of Features}

\section{1) Gradient Descriptors:}

Local Gradient Distribution: Gradient descriptors are considered among the most powerful descriptors [32]. The basic idea of most gradient descriptors is to accumulate a score for the orientation of local gradients [14], [33]. In this work, we adopt a modified version of Histogram of Oriented Gradients that accommodate for rotation-invariant properties [2]. Previously computed gradients $\mathbf{M}_{g}(x, y)$ of local cells of size $8 \times 8$ pixels are accumulated in nine-bin histograms according to their orientations $\mathbf{O}_{g}(x, y)$ after tri-linearly interpolating them both in position and orientation [14]. Then, cell histograms are circularly shifted using the global dominant orientation to achieve rotation-invariance properties.

$\mathbf{g}_{\text {cell }}(k)=\sum_{x, y} w\left(\mathbf{M}_{g}(x, y)\right) \mid \mathbf{O}_{g}(x, y) \in \mathbf{o}_{k}, k \in\{1,2, \ldots, 9\}$

where the weight of each vote $w$ is computed using tri-linear interpolation [34] in both position and orientation according to a system of equations that can be found in [34]. To achieve illumination invariance properties, the response of each square block of four cells $\mathbf{g}_{\text {block }}$ is locally normalised using $L 2$ normalisation with $\epsilon=0.05$. Blocks are overlapped resulting in 49 blocks of four cells, each is represented by 36 features. Responses from all normalised blocks $\mathbf{g}_{\text {block }} \leftarrow \frac{\mathbf{g}_{\text {block }}}{\sqrt{\left\|\mathbf{g}_{\text {block }}\right\|_{2}^{2}+\epsilon^{2}}}$ are concatenated in a single vector of 1764 features.

Global Gradient Distribution: In order to encode the global distribution of gradients, Fourier descriptors are exploited. They are used to capture spectral information (spectral information in an image is strongly related to the strength and the shape of edges). Subsequently, they can be used to depict global edge and gradient information as they do not preserve local spatial information. Furthermore, it can be used to differentiate rectilinear structures from other structures as an edge line in the spatial domain will appear as a line in the spectral domain with a 90 degree difference in orientation, as shown in Fig. 2. This is very useful for cars as they mainly consist of rectilinear structures as aforementioned. Fourier descriptors have been used in target detection with many variants and modifications [35], [36]. Nonetheless, the simplest form of the Fourier descriptors has been used in the proposed framework as it is the least computationally expensive.

The 2-D discrete Fourier Transform $\mathbf{F}(u, v)$ for an image $\mathbf{I}_{\text {grey }}$ of dimensions $X$ and $Y$ is defined in Equ. 16 [37]. To compute Fourier descriptors, detection windows are first rotated so that the dominant orientation will be vertical using the global orientation. By doing this, detection windows that have cars will have a vertical orientation. Rotated windows are then cropped keeping the central square region of $48 \times 48$ pixels such that extra padded parts, which are added because 


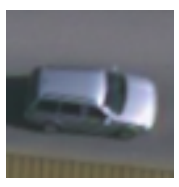

(a)

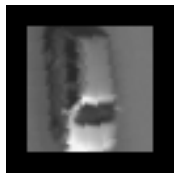

(e)

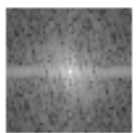

(i)

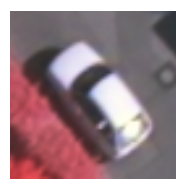

(b)

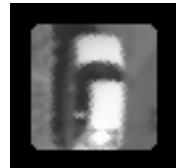

(f)

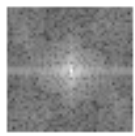

(j)

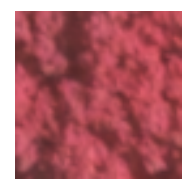

(c)

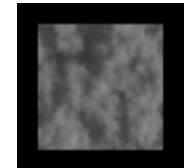

(g)

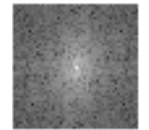

(k)

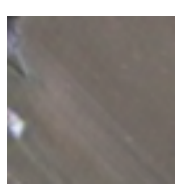

(d)

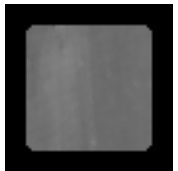

(h)

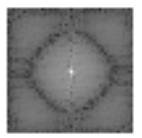

(1)
Fig. 2: Computation of the Fourier descriptor in target-class and non-targetclass patches: Target-class patches are shown in (a) and (b); their corresponding cropped rotated versions are shown in (e) and (f); their corresponding Fourier Transform coefficients are shown in (i) and (j), respectively. Nontarget-class patches are shown in (c) and (d); their corresponding cropped rotated versions are shown in (g), and (h); their corresponding Fourier Transform coefficients are shown in (k) and (l), respectively. Fourier Transform was computed after the rotation of the original patches so that the dominant orientation of the patches would be vertical. Shown images were scaled for viewing.

of the rotation process, are removed.

$$
\mathbf{F}(u, v)=\frac{1}{X Y} \sum_{x} \sum_{y} \mathbf{I}_{g r e y}(x, y) e^{-j 2 \pi\left(\frac{u x}{X}+\frac{v y}{Y}\right)}
$$

The Fourier feature vector $\mathbf{f}_{\text {window }}$ is formed by reshaping the matrix that contains the 2304 magnitudes of Fourier Transform coefficients into a single vector and it is then normalised using $L 2$ normalisation $\mathbf{f}_{\text {window }} \leftarrow \frac{\mathbf{f}_{\text {window }}}{\sqrt{\left\|\mathbf{f}_{\text {window }}\right\|_{2}^{2}+\epsilon^{2}}}$, with $\epsilon$ equals to 0.01 .

\section{2) Colour Descriptors:}

Local Colour Distribution: Combining the distribution of gradients with the distribution of colours will improve the performance of a particular target detector, if the target of interest possesses distinctive colour properties. For cars, they possess specific colour distribution: the colour of windscreens is always dark in aerial images and the colour of the car boot is the same as the colour of the car bonnet. Therefore, using a local Colour Self-Similarity (CSS) descriptor will encode the local similarity and variation of colours across pixels. An improved modified version of the colour self-similarity descriptors used by [38], [39] is proposed to complement HOG and Fourier descriptors and it is called truncated Pyramid Colour Self-Similarity (tPCSS). This new self-similarity descriptor $\mathbf{s}$ depends on the calculation of the intersection of local single-colour histograms to measure the similarity between two histograms $\mathbf{h}_{c}$ and $\mathbf{h}_{n}$ for the central cell and a neighbouring cell, respectively.

$$
\begin{aligned}
\mathbf{s}_{\text {cell }}=\left[\mathbf{h}_{c} \cap \mathbf{h}_{n}\right]= & {\left[\left\lceil\sum_{k} \min \left[\mathbf{h}_{c}(k), \mathbf{h}_{n}(k)\right]\right]\right], } \\
& n \in\{1,2, \ldots, 8\}, k \in\{1,2, \ldots, 16\}
\end{aligned}
$$

where $\lceil$.$\rceil indicates that the result is truncated at the size$ of an unsigned integer of eight bits, $n$ represents the $n^{\text {th }}$ neighbouring cell and $k$ represents the $k^{\text {th }}$ histogram bin. This truncation was found empirically to be necessary in order to have a high recall rate as it tolerates some variation among true-positive patches. The intersection is computed with the eight neighbouring cells. Local CSS descriptors are computed on a grey-scale version of the cropped rotated window on cells of $4 \times 4$ pixels and $8 \times 8$ pixels with overlapped neighbourhood. More specifically, in the first level of the pyramid structure, $8 \times 8$ pixel cells are used for the central $48 \times 48$ pixels, resulting in 16 central cells, each has eight features. Thus, the first level is represented by 128 features. As for the second level of the pyramid, $4 \times 4$ pixel cells are exploited. A total of 100 central cells are used, each has eight features. Hence, the second level is represented by 800 features. The final PCSS descriptor is formed by concatenating the $L 2$-normalised local responses from all the cells into a single vector of 928 features.

\section{Classification}

The proposed overall extended feature vector is formed by the concatenation of the computed ensemble of normalised descriptors: 1764 local gradient features, 2304 global gradient features and 928 local colour features. The total length of this new combined feature vector is 4996 features. Since the classification problem is characterised by having a relatively high dimensionality and following Occam's razor "Among competing hypotheses, the one with the fewest assumptions should be selected", a linear SVM classifier that is known to work efficiently in high-dimensional scenarios [3] has been adopted in the proposed framework. It is used to discriminate the car sub-space from the non-car sub-space with a regularisation parameter $c$ equals 15 . The SVM classifier is fed by the formed descriptor vector to distinguish between the car class sub-space and the non-car class sub-space.

In order to classify a new data sample $s$, the feature vector is computed using the transformation $\phi(s)$ that transforms this new data sample to a hyper-space of dimensionality $4996 \mathrm{D}$. The distance from the separating hyper-plane is calculated using the optimal weight $\mathbf{w}_{\text {opt }}$ and bias $b$. The sign of $z(s)$ determines on which side of the hyper-plane the data sample $s$ lies, i.e, to which sub-space the data sample belongs. The distance from the hyper-plane represents its confidence score. A detailed mathematical analysis of how $\mathbf{w}_{\text {opt }}$ and $b$ are obtained and how SVM classifier works can be found in [3].

$$
z(s)=\mathbf{w}_{o p t}^{T} \phi(s)+b
$$

\section{E. Post-processing}

The aim of the post-processing stage is to eliminate multiple detections per single true detection that are resulted from the 


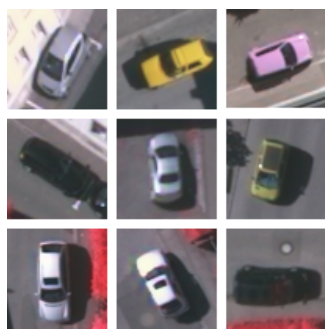

(a)

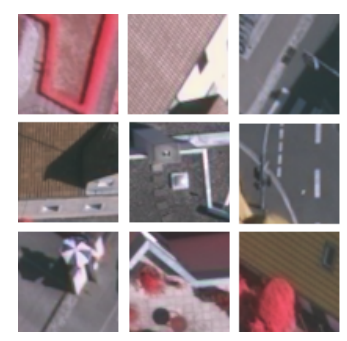

(b)
Fig. 3: Samples of the patches that were extracted from the Vaihingen dataset and were used for training: (a) positive training samples of car-class targets at different orientations and of various colours; (b) negative training samples of non-car-class targets. Shown images were scaled for viewing.

use of a sliding-window approach. Detection windows with positive score that overlap by more than $50 \%$ are eliminated except the one that has the highest output confidence score.

\section{DATASETS}

The Vaihingen dataset [29] was used for training both the GMM and the SVM classifiers. The Vaihingen dataset has a high resolution of ground sampling distance (GSD) equal to $8 \mathrm{~cm}$. It was captured over the city of Vaihingen - Germany by the German Society for Photogrammetry, Remote Sensing and Geoinformation (DGPF) [29]. The requirement that the viewpoint must be at a perpendicular or a slightly-oblique angle has been met. No pre-processing was performed on the dataset. The dataset is divided into two sub-sets by DGPF, one sub-set for training and the other for testing.

\section{A. Training Dataset}

Square patches of cars were taken from areas other than the test areas and were used as the positive training dataset. They were taken from various regions such that a great diversity in car orientations and backgrounds could be achieved. The patches were cropped such that there were at least six pixels around the body of each car so that gradients could be efficiently computed and then patches were rescaled to a size of $64 \times 64$ pixels. Mirrored and horizontally-flipped versions of these square patches were included to make the dataset richer in the diversity among cars' orientations. For negative training data, random patches were selected from different backgrounds as well as bootstrapped hard negatives that were fired as false positives during validation, which was carried on the training dataset. A total of 754 positive patches and 3601 negative patches were selected. Sample patches are shown in Fig. 3.

\section{B. Testing Dataset}

The proposed car detection framework was tested on the test dataset specified by DGPF. It contains typical urban backgrounds with different building styles, roads and vegetation. More specifically, the dataset contains dense development consisting of historic buildings having complex shapes, highrising residential buildings that are surrounded by trees and pure residential areas with small detached houses.

\section{EXPERIMENTAL RESULTS}

The proposed framework was run on the test areas of the Vaihingen and such data was not included in the data over which the SVM classifier was trained. The performance was assessed and evaluated using qualitative analysis by visual inspection of the bounding boxes that were produced as a result of the application of the proposed framework on the data. In addition, various quantitative measures, namely, the precisionrecall rates, the average precision and the interpolated average precision, were used.

\section{A. Qualitative Analysis}

Visual results of the experiments of applying the proposed framework on sample regions of the test areas of the Vaihingen dataset are shown in Fig. 4. The proposed framework is able to detect successfully cars in typical urban scenes with different colours and at different orientations with a high recall rate and a very low false-positive rate. It can be observed from the shown binary masks in Fig. 4 that the extraction of regions of interest is approximate. For example, any area that is overimposed by shadows was classified as a region of interest as such areas possess a similar colour distribution to that of road and parking areas. Nevertheless, this approximate extraction is effective in eliminating areas that contain buildings and vegetation and consequently this step helps reduce the falsepositive rate.

\section{B. Quantitative Analysis}

Quantitative evaluation methods for target detection systems depend on the adopted approach [40]. As a window-based approach was adopted alongside a classifier that outputs a confidence score, two widely-used metrics were used to evaluate the performance and to compare it to the performance of the state of the art that constitutes the baseline performance. These metrics are the average precision $A P$ and the interpolated average precision $A P_{\text {inter }}$. Both metrics depend on ranked outputs according to the confidence score in a descending order.

In the adopted evaluation methodology, a detected bounding box $B_{D}$ was considered true if it matched a corresponding ground truth bounding box $B_{G T}$ using Equ. 19 and there was no other bounding boxes matching the same ground truth [41]. In the case of having more than one bounding box matching the same ground truth, only one of them was considered a true detection and others were considered false positives. Ambiguous objects, for example objects which are partially occluded were not taken into account, when measuring the performance of the proposed framework as in [16], [17], [19].

$$
\frac{\operatorname{Area}\left(B_{D} \cap B_{G T}\right)}{\operatorname{Area}\left(B_{D} \cup B_{G T}\right)} \geq 0.5
$$

After defining the condition for considering true positives, precision $P R$ and recall $R C$ can be computed at several levels according to the rank of the confidence score associated with a detection window using Equ. 20 and Equ. 21, respectively. The term "recall" is used here to indicate the proportion of the truly-detected cars to the total number of ground-truth cars, 


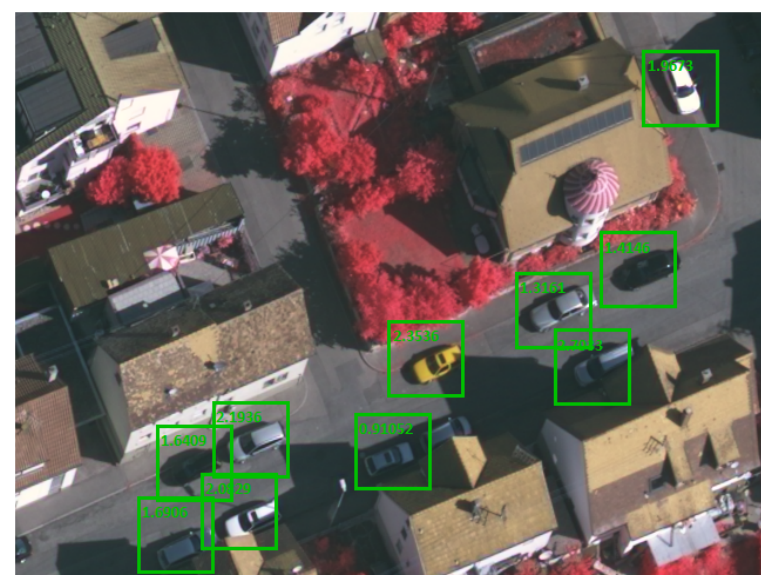

(a)

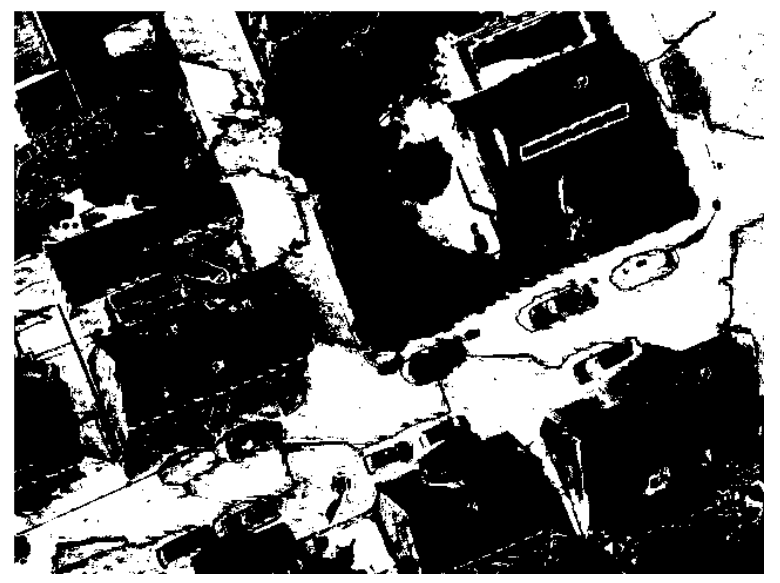

(c)

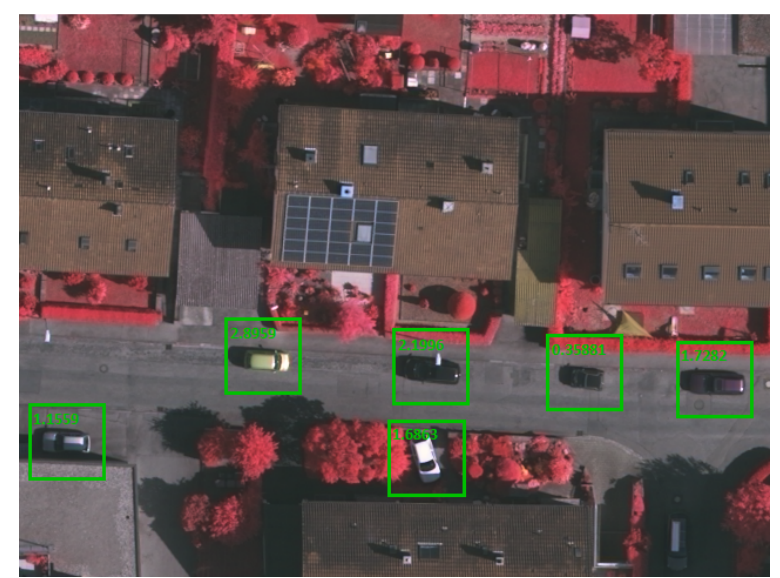

(b)

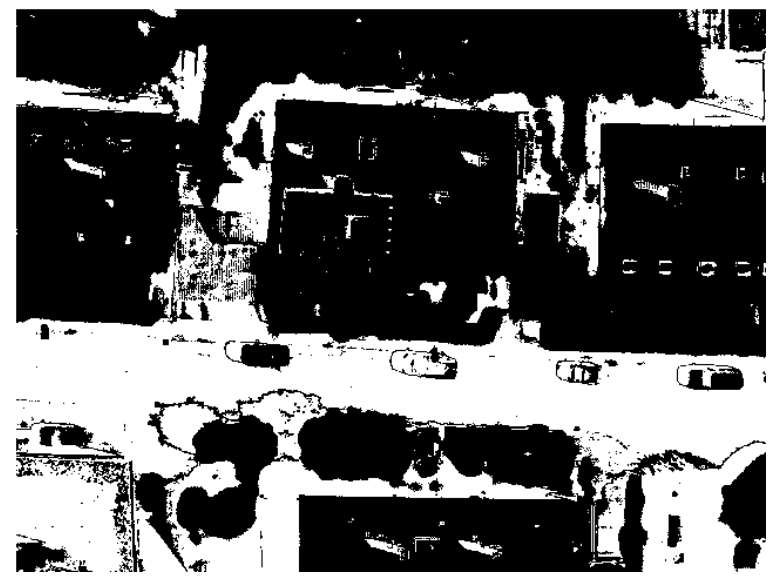

(d)

Fig. 4: Sample regions of the test areas of the Vaihingen dataset after applying the proposed car-detection framework: (a) and (b) outputs of the the proposed framework, bounding boxes represent detections and the number at the top of each bounding box represents its confidence score; (c) and (d) binary masks produced by the GMM classifier. Shown images were scaled for viewing.

whereas the term "precision" indicates the proportion of trulydetected cars to the total number of detections above a given confidence score [41].

$$
\begin{gathered}
P R=\frac{\# \mathbf{b}_{T P}}{\# \mathbf{b}_{T P}+\mathbf{b}_{T P}} \\
R C=\frac{\# \mathbf{b}_{T P}}{\# \mathbf{b}_{G T}}
\end{gathered}
$$

where $\mathbf{b}_{T P}$ represents true positives, $\mathbf{b}_{F P}$ represents false positives and $\mathbf{b}_{G T}$ represents ground truth.

Fig. 5 shows the obtained precision-recall curves of the proposed framework. It also compares the obtained results of the proposed framework with the results of the baseline frameworks. It can be deduced that for the same recall rate, the proposed framework achieves a higher precision rate than that of the works in [2], [17], [26] with their default settings. It is worth to mention that in our implementation to the work in [26], we used a final non-maximum suppression stage as the original method produces multiple detections per single true detection. In addition, this allows to compare our proposed features with feature-extraction methods using deep convolutional neural networks. All baseline frameworks were trained and tested on the Vaihingen dataset.

The second metric that is used to evaluate the performance of the proposed framework is the average precision $A P$. Another version of the $A P$ is the interpolated average precision $A P_{\text {inter }}$. It is worth to mention that the $A P_{\text {inter }}$ penalises methods that are not able to retrieve $100 \%$ of the ground truth data [41], unlike the $A P$ that does not take this into account.

$$
\begin{gathered}
A P=\frac{1}{\# \mathbf{b}_{T P}} \sum_{k \in\left\{1,2, \ldots, \# \mathbf{b}_{T P}\right\}} \mathbf{P R}\left(\mathbf{b}_{T P}(k)\right) \\
A P_{\text {inter }}=\frac{1}{11} \sum_{k \in\{0,0.1, \ldots, 1\}} \mathbf{P R}_{\text {interp }}(k) \mid \mathbf{P R}_{\text {interp }}(k)= \\
\max _{\tilde{r}: \tilde{r} \geq r}[\mathbf{P R}(\tilde{r})]
\end{gathered}
$$

where $\mathbf{b}_{T P}$ represents true positives at a given confidence score and PR represents the precision. Table I compares the performance of proposed framework and baseline methods. Clearly, the proposed framework outperforms the state of the art by achieving a higher $A P_{\text {inter }}$ on the Vaihingen dataset using a linear classifier as opposed to the non-linear classifier 


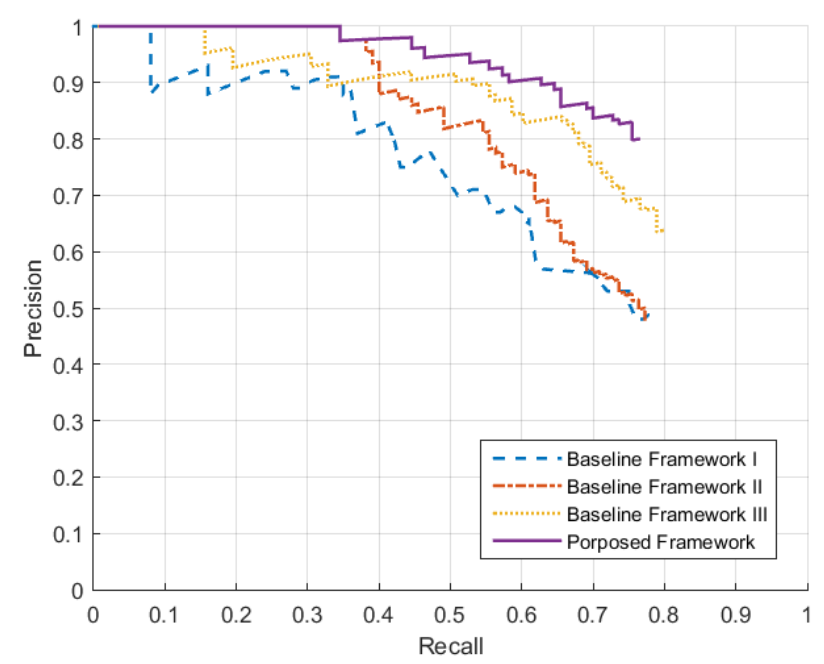

Fig. 5: Performance comparison among the proposed framework and baseline methods on the Vaihingen dataset represented by precision rates at different recall rates: baseline framework I is the work of [17], baseline framework II is the work of [2] and baseline framework III is the work of [26].

used in [17] and the deep model used in [26] and despite the fact that this metric penalises methods that retrieve only a sub-set of the ground truth [41].

In order to analyse the performance of the proposed framework and get a better understanding of how each stage contributes to the overall performance, the performance of the ensemble of descriptors in conjunction with the SVM classifier was assessed on the testing dataset. In addition, the performance was assessed with adding only one of the proposed window filtering steps. Fig. 6 and Table II demonstrate the obtained results under six different scenarios: i) ensemble of descriptors is used in conjunction with the SVM classifier only, ii) window evaluation based on the difference in grey histograms and the ensemble of descriptors are used in conjunction with the SVM classifier, iii) window evaluation based on the level of texture and the ensemble of descriptors are used in conjunction with the SVM classifier, iv) window evaluation based on the value of the maximum eigenvalue and the ensemble of descriptors are used in conjunction with the SVM classifier, v) window evaluation based on the amount of overlap with the regions of interest and the ensemble of descriptors are used in conjunction with the SVM classifier and vi) the overall proposed framework.

It can be inferred that the three-step window filtering process has a slight effect on reducing false positives as compared to the effect of the extraction-of-regions-of-interest stage. Nonetheless, the window-filtering process has a significant effect on reducing the number of detection windows that will be passed to the following stages, as it can be seen in Table II. More specifically, incorporating either the new histogram difference or texture checks leads to reducing the number of detection windows to almost half. The table also shows that evaluating windows based on the amount of overlap with the extracted of regions of interest

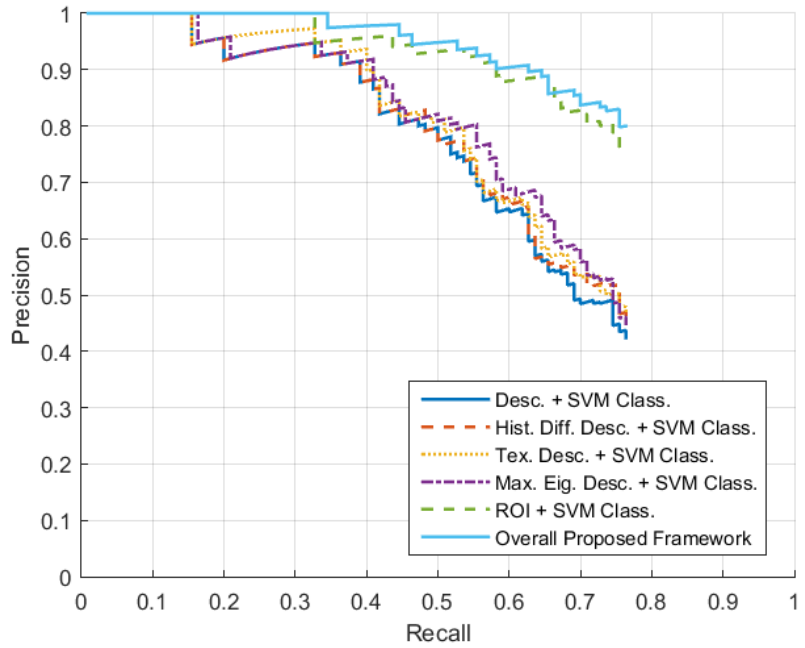

Fig. 6: Performance of the proposed framework on the Vaihingen dataset represented by precision rates at different recall rates: (i) obtained precisionrecall curve when the descriptors were used alongside the SVM classifier; (ii) obtained precision-recall curve when the histogram-difference check was used in conjunction with the descriptors and the SVM classifier; (iii) obtained precision-recall curve when the texture check was used in conjunction with the descriptors and the SVM classifier; (iv) obtained precision-recall curve when the maximum-eigenvalue check was used in conjunction with the descriptors and the SVM classifier; (v) obtained precision-recall curve when the regions-of-interest check was used in conjunction with the descriptors and the SVM classifier; (vi) obtained precision-recall curve of the overall proposed framework.

TABLE I: Performance comparison among the proposed framework and baseline methods on the Vaihingen dataset represented by the average precision $A P$ and the interpolated average precision $A P_{\text {inter: }}$ : baseline framework I is the work of [17], baseline framework II is the work of [2] and baseline framework III is the work of [26].

\begin{tabular}{|c|c|c|}
\hline & $A P$ & $A P_{\text {inter }}$ \\
\hline Baseline Framework I & $77.81 \%$ & $58.92 \%$ \\
\hline Baseline Framework II & $86.49 \%$ & $64.26 \%$ \\
\hline Baseline Framework II & $89.54 \%$ & $66.44 \%$ \\
\hline Proposed Framework & $\mathbf{9 5 . 5 1} \%$ & $\mathbf{6 9 . 8 7 \%}$ \\
\hline
\end{tabular}

has a significant effect on increasing the overall precision rate.

Accuracy of the Estimation of the Orientation of Cars: The proposed framework uses the eigenvalues of the covariance matrix of the local distribution of gradients to estimate the dominant orientation of cars as previously discussed. The accuracy of estimating the dominant orientation is $98.34 \%$.

\section{Running-Time Analysis}

The proposed framework has been implemented in a MAT$\mathrm{LAB}$ environment and all experiments were run on an IntelCore-i5 CPU with speed of $2.5 \mathrm{GHz}$ and a Random Access Memory of $6.00 \mathrm{~GB}$ size. Whilst the machine that was used has multiple processing cores, the current implementation of the framework uses only a single core.

Table III shows the number of detection windows that can be processed per second under different settings. The overall proposed framework is able to process 136 windows per second $(0.035 \mathrm{MB})$. This indicates that the current implementation 
TABLE II: Performance of the proposed framework with six different settings on the Vaihingen dataset represented by the average precision $A P$, the interpolated average precision $A P_{\text {inter }}$ and the percentage of the detection windows that were passed to the SVM classifier.

\begin{tabular}{|c|c|c|c|}
\hline & $A P$ & $A P_{\text {inter }}$ & Windows \\
\hline Desc. + SVM Class. & $81.86 \%$ & $61.14 \%$ & $100.00 \%$ \\
\hline Hist. Diff. + Desc. + SVM Class. & $82.67 \%$ & $61.73 \%$ & $50.14 \%$ \\
\hline Text. + Desc. + SVM Class. & $83.85 \%$ & $62.53 \%$ & $51.36 \%$ \\
\hline Max. Eig. + Desc. + SVM Class. & $84.37 \%$ & $62.81 \%$ & $71.35 \%$ \\
\hline ROI + Desc. + SVM Class. & $94.31 \%$ & $69.06 \%$ & $48.45 \%$ \\
\hline All + Desc. + SVM Class. & $\mathbf{9 5 . 5 1} \%$ & $\mathbf{6 9 . 8 7 \%}$ & $\mathbf{1 2 . 0 4 \%}$ \\
\hline
\end{tabular}

TABLE III: Running time analysis of the proposed framework with six different settings represented by the number windows that can be processed per second and the size in MB that can be processed per second

\begin{tabular}{|c|c|c|}
\hline & Windows/s & $\mathrm{MB} / \mathrm{s}$ \\
\hline Desc. + SVM Class. & 29 & 0.008 \\
\hline Hist. Diff. + Desc. + SVM Class. & 49 & 0.012 \\
\hline Text. + Desc. + SVM Class. & 52 & 0.013 \\
\hline Max. Eig. + Desc. + SVM Class. & 52 & 0.013 \\
\hline ROI + Desc. + SVM Class. & 46 & 0.012 \\
\hline All + Desc.+ SVM Class. & $\mathbf{1 3 6}$ & $\mathbf{0 . 0 3 5}$ \\
\hline
\end{tabular}

has a near-real-time performance in a MATLAB environment that is generally known to be slow.

\section{CONCLUSION}

This paper presents a novel framework for the detection of cars in high-resolution aerial images of complex urban environments scenes. The proposed framework consists of two main sub-systems: window evaluation and window classification. The performance of the proposed framework was assessed on the challenging Vaihingen dataset using the precision-recall curves, the average precision and the interpolated average precision. The results clearly demonstrated the effectiveness of the proposed framework in detecting cars with high precision in complex urban environments and most importantly they corroborated its superiority to the state of the art.

Several points can be learnt from the implementation and the results of the proposed framework. In heavily-cluttered environments, e.g., urban environments, the existence of many objects that are visually similar to cars can produce a high rate of false positives. Some previous works have tackled this problem by using road maps to narrow down search areas to roads. The problem with this approach is that it requires the knowledge of road maps prior to the detection process and the use of a map-projection method, which is computationally expensive. In the proposed framework, it was shown that an approximate extraction of roads can be achieved using a Gaussian Mixture Model that is taught to distinguish pixels based on colours. Added to that, it can be inferred that the local distributions of gradients and colours play an important role in describing a specific target of interest. The dominant orientation of detection windows can be accurately estimated using the order of the maximum eigenvalue of the covariance matrix of locally-accumulated gradients. Designing a window-evaluation stage helps reduce the processing time.
Furthermore, it can be concluded that the optimal choice of image cues to be used to depict a particular class of object plays an important role in the achievement of improved performance even in heavily-cluttered environments using lowdimensional feature vectors and linear classification.

Among the possible extensions to the proposed framework is the modification of the image descriptors that have been used, the combination of more image descriptors, the development of novel descriptors and the use of dimensionality reduction techniques. The devised image descriptors were designed for the purpose of the detection of cars in airborne imagery. Nevertheless, they have the potential to be generalised and their application to detect other non-car targets can be investigated.

\section{MATHEMATICAL NotaTion}

$\begin{array}{ll}X, x & \text { Scalar } \\ \mathbf{x} & \text { Column Vector } \\ {\left[x_{1}, \ldots, x_{K}\right]} & \text { Row Vector } \\ (.)^{T} & \text { Transpose Operator } \\ (.)^{H} & \text { Hermitian Transpose Operator } \\ \lceil.\rceil & \text { Truncation Operator } \\ \mathbf{X} & \text { Matrix } \\ y(x) & \text { Function } \\ \mathbb{E}\{.\} & \text { Expectation Operator } \\ \mathbb{R} & \text { Covariance Matrix across } \mathbf{x} \\ \# \mathbf{x} & \text { Cardinality of } \mathbf{x} \\ d & \text { Eigenvalue } \\ D & \text { Dimensionality }\end{array}$

\section{REFERENCES}

[1] "The earth observation handbook," 2012.

[2] M. ElMikaty and T. Stathaki, "Car detection in high-resolution urban scenes using multiple image descriptors," in IAPR International Conference on Pattern Recognition, 2014, pp. 4299-4304.

[3] C. M. Bishop, Pattern Recognition and Machine Learning, Springer, 2006.

[4] V. Ramakrishnan, A. K. Prabhavathy, and J. Devishree, "A survey on vehicle detection techniques in aerial surveillance," International Journal of Computer Applications, vol. 55, no. 18, pp. 43-47, 2012.

[5] M. Liu, Y. Wu, P. Zhang, Q. Zhang, Y. Li, and M. Li, "Sar target configuration recognition using locality preserving property and gaussian mixture distribution," IEEE Geoscience and Remote Sensing Letters, vol. 10, no. 2, pp. 268-272, 2013.

[6] X. Yuan, T. Tang, D. Xiang, Y. Li, and Y/ Su, "Target recognition in sar imagery based on local gradient ratio pattern," International Journal of Remote Sensing, vol. 35, no. 3, pp. 857-870, 2014.

[7] T. Zhao and R. Nevatia, "Car detection in low resolution aerial image," in IEEE International Conference on Computer Vision, 2001, vol. 1, pp. 710-717 vol.1.

[8] S. Hinz, "Detection and counting of cars in aerial images," in IEEE International Conference on Image Processing, 2003, vol. 3, pp. 9971000 . 
[9] S. Hinz, C. Schlosser, and J. Reitberger, "Automatic car detection in high resolution urban scenes based on an adaptive 3D-model," in GRSS/ISPRS Joint Workshop on Remote Sensing and Data Fusion over Urban Areas, 2003, pp. 167-171.

[10] J. Choi and Y. Yang, "Vehicle detection from aerial images using local shape information," in Advances in Image and Video Technology, vol. 5414 of Lecture Notes in Computer Science, pp. 227-236. Springer, 2009.

[11] D. Comaniciu and P. Meer, "Mean shift analysis and applications," in IEEE International Conference on Computer Vision, 1999, vol. 2, pp. 1197-1203 vol.2.

[12] T. Nguyen, H. Grabner, H. Bischof, and B. Gruber, "On-line boosting for car detection from aerial images," in IEEE International Conference on Research, Innovation and Vision for the Future, 2007, pp. 87-95.

[13] P. Viola and M. Jones, "Robust real-time face detection," in IEEE International Conference on Computer Vision, 2001, vol. 2, pp. 747747.

[14] N. Dalal and B.Triggs, "Histograms of oriented gradients for human detection," in IEEE Conference on Computer Vision and Pattern Recognition, 2005, vol. 1, pp. 886-893.

[15] T. Ojala, M. Pietikainen, and T. Maenpaa, "Multiresolution gray-scale and rotation invariant texture classification with local binary patterns," IEEE Transactions on Pattern Analysis and Machine Intelligence, vol. 24, no. 7, pp. 971-987, 2002 .

[16] A. Kembhavi, D. Harwood, and L.S. Davis, "Vehicle detection using partial least squares," IEEE Transactions on Pattern Analysis and Machine Intelligence, vol. 33, no. 6, pp. 1250-1265, 2011.

[17] W. Shao, W. Yang, G. Liu, and J. Liu, "Car detection from highresolution aerial imagery using multiple features," in IEEE International Geoscience and Remote Sensing Symposium, 2012, pp. 4379-4382.

[18] Sebastien S. Razakarivony and F. Jurie, "Small target detection combining foreground and background manifolds," in IAPR International Conference on Machine Vision Applications, 2013, p. 4.

[19] S. Madhogaria, P. M. Baggenstoss, M. Schikora, W. Koch, and D. Cremers, "Car detection by fusion of HOG and causal MRF," IEEE Transactions on Aerospace and Electronic Systems, vol. 51, no. 1, pp. 575-590, 2015.

[20] H. Moon, R. Chellappa, and A. Rosenfeld, "Optimal edge-based shape detection," IEEE Transactions on Image Processing, vol. 11, no. 11, pp. $1209-1227,2002$.

[21] H. Moon, R. Chellappa, and A. Rosenfeld, "Performance analysis of a simple vehicle detection algorithm," Image and Vision Computing, vol. 20, no. 1, pp. 1-13, 2002.

[22] H. Zheng, L. Pan, and L. Li, "A morphological neural network approach for vehicle detection from high resolution satellite imagery," in Neural Information Processing, vol. 4233 of Lecture Notes in Computer Science, pp. 99-106. Springer Berlin Heidelberg, 2006.

[23] L. Eikvil, L. Aurdal, and H. Koren, "Classification-based vehicle detection in high-resolution satellite images," ISPRS Journal of Photogrammetry and Remote Sensing, vol. 64, no. 1, pp. 65 - 72, 2009.

[24] S. Tuermer, F. Kurz, P. Reinartz, and U. Stilla, "Airborne vehicle detection in dense urban areas using hog features and disparity maps," IEEE Journal of Selected Topics in Applied Earth Observations and Remote Sensing, , no. 99, pp. 1-11, 2013.

[25] X. Chen, S. Xiang, C. L. Liu, and C. H. Pan, "Vehicle detection in satellite images by hybrid deep convolutional neural networks," IEEE Geoscience and Remote Sensing Letters, vol. 11, no. 10, pp. 1797-1801, 2014.

[26] M. Yi, F. Yang, E. Blasch, C. Sheaff, K. Liu, G. Chen, and H. Ling, "Vehicle classification in WAMI imagery using deep network," in Proceedings of SPIE on Sensors and Systems for Space Applications IX, 2016, vol. 9838, p. 98380.

[27] A. Krizhevsky, I. Sutskever, and G. E. Hinton, "Imagenet classification with deep convolutional neural networks," in Advances in Neural Information Processing Systems, pp. 1097-1105. Curran Associates, Inc., 2012

[28] J. Canny, "A computational approach to edge detection," IEEE Transactions on Pattern Analysis and Machine Intelligence, vol. 8, no. 6, pp. 679-698, 1986.

[29] M. Cramer, "The DGPF-test on digital airborne camera evaluationoverview and test design," Photogrammetrie-FernerkundungGeoinformation, vol. 2, no. 2, pp. 73-82, 2010.

[30] G. McLachlan and D. Peel, Finite Mixture Models, Wiley, 2000.

[31] B. Alexe, T. Deselaers, and V. Ferrari, "Measuring the objectness of image windows," IEEE Transactions on Pattern Analysis and Machine Intelligence, vol. 34, no. 11, pp. 2189-2202, 2012
[32] P. Dollar, C. Wojek, B. Schiele, and P. Perona, "Pedestrian detection An evaluation of the state of the art," IEEE Transactions on Pattern Analysis and Machine Intelligence, vol. 34, no. 4, pp. 743-761, 2012.

[33] D. G. Lowe, "Object recognition from local scale-invariant features," in IEEE International Conference on Computer Vision, 1999, vol. 2, pp. $1150-1157$.

[34] N. Dalal, Finding People in Images and Videos, Ph.D. thesis, Institut Polytechnique de Grenoble, 2006.

[35] J.W. Gorman, O.R. Mitchell, and F.P. Kuhl, "Partial shape recognition using dynamic programming," IEEE Transactions on Pattern Analysis and Machine Intelligence, vol. 10, no. 2, 1988.

[36] E. Mavridou, H. Manh-Dung, J.L. Crowley, and A. Lux, "Scale normalized radial fourier transform as a robust image descriptor," in IAPR International Conference on Pattern Recognition, 2014, pp. 39933998.

[37] M. Sonka, V. Hlavac, and R. Boyle, Image Processing, Analysis, and Machine Vision, Thomson Learning, 3rd edition, 2007.

[38] S. Walk, N. Majer, K. Schindler, and B. Schiele, "New features and insights for pedestrian detection," in IEEE Conference on Computer Vision and Pattern Recognition, 2010, pp. 1030-1037.

[39] N. Wang, X. Gong, and J. Liu, "A new depth descriptor for pedestrian detection in rgb-d images," in IAPR International Conference on Pattern Recognition, 2012, pp. 3688-3691.

[40] V. Y. Mariano, M. Junghye, P. Jin-Hyeong, R. Kasturi, D. Mihalcik, H. Li, D. Doermann, and T. Drayer, "Performance evaluation of object detection algorithms," in IAPR International Conference on Pattern Recognition, 2002, vol. 3, pp. 965-969 vol.3.

[41] M. Everingham, L. Van Gool, C. K. I. Williams, J. Winn, and A. Zisserman, "The PASCAL Visual Object Classes (VOC) challenge," International Journal of Computer Vision, vol. 88, no. 2, pp. 303-338, 2010.

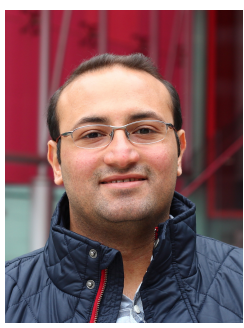

Mohamed EIMikaty is a Research Associate at the Department of Electrical and Electronic Engineering, Imperial College London, U.K. He was awarded the B.Sc. degree in Electronics and Communications Engineering with honours from Mansoura University, Egypt, in 2009, the M.Sc. degree in Communications and Signal Processing Engineering with distinction from Imperial College London, UK, in 2012 and the Ph.D. degree in Electrical and Electronic Engineering from Imperial College London, UK, in 2016.

$\mathrm{He}$ has been engaged in various industrial research projects in Egypt and the UK. His current research interests include Image Processing and Computer Vision with emphasis on Image Representation, Image Analysis, Image Understanding techniques with applications to object detection, recognition and tracking.

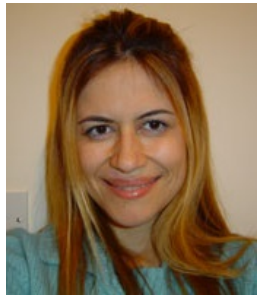

Tania Stathaki was born in Athens, Greece. She received the Masters degree in Electronics and Computer Engineering from the Department of Electrical and Computer Engineering, National Technical University of Athens, Athens, Greece, and the Ph.D. degree in signal processing from Imperial College, London, U.K. She was a Lecturer with the Department of Information Systems and Computing, Brunel University, London, U.K., and an Assistant Professor with the Department of Technology Education and Digital Systems, University of Piraeus, Piraeus, Greece. She is currently a Reader with the Department of Electrical and Electronic Engineering, Imperial College.

She has intensive research experience in image processing and computer vision and, more specifically, image fusion, image registration, change detection, object detection and recognition, and object tracking. She has mainly been involved in defence and security applications and has collaborated with various UK companies such as Dstl, General Dynamics, Selex Galileo and BAE Systems. She is actively involved in various European Union and UK research projects. She has authored or co-authored many papers on signal and image processing and computer vision. 